\title{
Polarization of accreting X-ray pulsars - II. Hercules X-1
}

\author{
Ilaria Caiazzo ${ }^{\oplus 1,2 \star}$ and Jeremy Heyl ${ }^{2}$ \\ ${ }^{1}$ TAPIR, Walter Burke Institute for Theoretical Physics, Mail Code 350-17, Caltech, Pasadena, CA 91125, USA \\ ${ }^{2}$ Department of Physics and Astronomy, University of British Columbia, Vancouver, BC V6T 1Z1, Canada
}

Accepted 2020 October 29. Received 2020 October 29; in original form 2020 May 27

\begin{abstract}
We employ our new model for the polarized emission of accreting X-ray pulsars to describe the emission from the luminous X-ray pulsar Hercules X-1. In contrast with previous works, our model predicts the polarization parameters independently of spectral formation, and considers the structure and dynamics of the accretion column, as well as the additional effects on propagation due to general relativity and quantum electrodynamics. We find that our model can describe the observed pulse fraction and the pulse shape of the main peak, as well as the modulation of the cyclotron line with phase. We pick two geometries, assuming a single accretion column or two columns at the magnetic poles, that can describe current observations of pulse shape and cyclotron modulation with phase. Both models predict a high polarization fraction, between 60 and 80 percent in the 1-10 keV range, that is phase and energy dependent, and that peaks at the same phase as the intensity. The phase and energy dependence of the polarization fraction and of the polarization angle can help discern between the different geometries.
\end{abstract}

Key words: accretion, accretion discs - polarization-relativistic processes - scattering-pulsars: individual: Hercules X-1 - Xrays: binaries.

\section{INTRODUCTION}

The polarization of light provides two observables, polarization degree and angle, that are very sensitive to the anisotropies of the emission region; therefore, polarization, combined with spectral and timing analysis, can be a powerful tool to study the geometry of astronomical sources. Regular astrophysical observations of X-ray polarization will soon become a reality, as several observatories with an X-ray polarimeter onboard are now being developed. The NASA SMEX mission IXPE (Weisskopf et al. 2016), in the 1-10 keV energy range, and the Indian POLIX (5-30 keV), are both scheduled to launch in 2021, as well as the rocket-based REDSOx $(0.2-0.8 \mathrm{keV}$; Gaenther et al. 2017), while the balloon-borne $X$-Calibur (Beilicke et al. 2014) and $P o G O+$ (Chauvin et al. 2018) are already flying. Additionally, the Chinese-European eXTP (Zhang et al. 2016; 1$10 \mathrm{keV}$ ), is scheduled for launch in 2025, while the narrow-band $(250 \mathrm{eV})$ LAMP (She et al. 2015) and the broad-band (0.2-60 keV) $X P P$ (Jahoda et al. 2019; Krawczynski et al. 2019) are still at the concept stage. Many of these upcoming polarimeters employ the gas pixel detector technology, or GPD, which was recently put to test by the CubeSat PolarLight (Feng et al. 2019), a small polarimeter without optics, that was able to measure the polarization of the Crab nebula (Feng et al. 2020).

As always when a new window opens in astronomy, it is fair to expect that the new polarimetric capabilities of the upcoming telescopes in the X-rays will lead to new breakthroughs in the study of X-ray sources, in particular for accreting neutron stars and black holes. In order to reap the most from these future observations, we need to be ready with a suite of theoretical models that can constrain,

^E-mail: ilariac@ caltech.edu when compared with observations, what is still unknown about these objects. In this paper, which is the second in a series, we present a method for modelling the polarized emission of accreting X-ray pulsars that agrees with current spectroscopic observations and that makes clear predictions of the polarization parameters depending on the geometry of the accretion region and on the strength of the magnetic field. In the previous paper (Caiazzo \& Heyl 2020, hereafter Paper I), we described in detail the model, whereas here we apply our method to model the polarized emission from the accreting X-ray pulsar Hercules X-1 (Her X-1).

Accreting X-ray pulsars are neutron stars that live in a close binary and accrete material from a companion star. They usually have high magnetic fields, of the order of $10^{12}-10^{13} \mathrm{G}$. For this reason, the accretion disc is truncated at the magnetospheric radius, several thousand kilometres from the neutron star, where the magnetic pressure from the star's dipolar magnetic field and the ram pressure in the disc become comparable, and the ionized gas is then funnelled to the magnetic poles of the star along the magnetic field lines. Depending on the luminosity of the radiation at the magnetic poles, the ionized gas may be able to reach the surface of the neutron star, heating the magnetic pole and creating hotspots, or the escaping photons may be able to slow down the in-flowing gas before it reaches the surface, and therefore an accretion column may form above the pole, held together by magnetic pressure. In both cases, the small area of the emission region coupled with the rotation of the star gives origin to the pulsating nature of the emission.

Her X-1 was one of the first accreting X-ray pulsars to be discovered (Tananbaum et al. 1972) and is one of the brightest and most studied; for this reason, it will be one of the main targets of all the upcoming polarimeters. Her X-1 is a persistent source in the $\mathrm{X}$-rays, and its X-ray flux shows several periodic modulations. The 
neutron star is orbiting a $2.2 \mathrm{M}_{\odot}$ stellar companion (Reynolds et al. 1997), HZ Her, with a period of 1.7 d, as can be inferred from the deep eclipses in the X-ray flux, while the spin period of the neutron star itself is $1.24 \mathrm{~s}$. A third, superorbital modulation, thought to be caused by the precession of the accretion disc, has a period of $35 \mathrm{~d}$ (Giacconi et al. 1973). The 35 d cycle shows two 'on' states, in which the pulsed emission is observed: a 'Main-On' of about $10 \mathrm{~d}$ and a 5-d 'Short-On', separated by two 'off' states during which the emission from the neutron star is occulted by the disc (Gerend \& Boynton 1976; Boynton, Crosa \& Deeter 1980; Scott \& Leahy 1999; Scott, Leahy \& Wilson 2000; Klochkov et al. 2008; Vasco et al. 2013).

Intriguingly, the pulse profile evolves following the same 35$\mathrm{d}$ cycle, with features appearing and disappearing with phase and energy (Truemper et al. 1986; Deeter et al. 1998; Scott et al. 2000; Staubert et al. 2013). This modulation in the pulse shape has been explained by the precession of the neutron star (Truemper et al. 1986; Postnov et al. 2013) or of the disc (Scott et al. 2000; Leahy 2002). Both theories present some issues. The neutron star precession model implies that a very strong and not yet identified feedback mechanism links the clock of the disc's and the neutron star's precession, as the two appear perfectly synchronized on very short time-scales; additionally, a mechanism is needed to explain why the precession period of the neutron star changes every few years by a few per cent. On the other hand, if the appearing and disappearing of features are to be explained by the occultations of a precessing disc, the disc has to be very close to the star, at about 20-40 neutron star radii, which is more than 10 times closer than the magnetospheric radius for a magnetic field of a few $10^{12} \mathrm{G}$. For a detailed discussion, see Staubert et al. (2013). Here, we suggest an alternative explanation, in which the features in the pulse profile that show a modulation in the 35-d period are caused by reflections off the precessing disc of the beamed radiation coming from the neutron star (see Section 3).

The modulations of spectral features can provide further insights on the geometry of the source and on the origin of the emission. The spectra of many accreting X-ray pulsars show cyclotron resonant scattering features (CRSFs), caused by resonant scattering of photons off electrons in the high magnetic field (Truemper et al. 1978; ArayaGóchez \& Harding 2000). The CRSFs provide a direct measurement of the strength of the magnetic field in the emission region, and that is how the magnetic field of Her X-1 has been measured to be a few times $10^{12} \mathrm{G}$. The cyclotron feature's energy is often modulated with rotational phase, and this has been explained by the fact that as the star rotates, the emission that we see comes from different emitting spots, where the magnetic field has different strengths (e.g. in Kreykenbohm et al. 2004). In some pulsars, the CRSF shows a long-term evolution as well that is thought to be due to changes in the geometry of the accretion region or of the local magnetic configuration (Becker et al. 2012; Nishimura 2014; Staubert et al. 2014). In particular, the centroid energy of the CRSF in Her X-1 has shown a steady decline until the year 2010 and then it has remained stable (Ji et al. 2019, and references therein). Some sources show an additional long-term variability in the cyclotron line scattering feature that correlates (or anticorrelates) with accretion luminosity (Tsygankov et al. 2006). As the pulsars that show a negative correlation are also the brightest ones, it has been suggested that a negative correlation is also an indication of supercritical luminosity and therefore the difference in variability could be caused by a difference in accretion geometry (Poutanen et al. 2013; Lutovinov et al. 2015; Mushtukov et al. 2015, 2018).

Another spectral feature that is also seen varying with the pulse phase is the iron $\mathrm{K} \alpha$ emission line at about $6.4 \mathrm{keV}$, which is thought to be caused by fluorescence in cool gas illuminated by an X-ray beam. The location of this material is not certain, as it can be the accretion disc, a corona above it, or even the atmosphere of the companion star (Choi et al. 1994), but the inner edge of the accretion disc seems to be the most likely location. Using RXTE PCA data on a 'Main-On' state, Vasco et al. (2013) presented a deep analysis of the evolution of spectral parameters with spin phase and with precession phase. Their findings were later confirmed by a study performed with NUSTAR data by Fürst et al. (2013).

We here apply the method developed in Paper I to reproduce many of the observational features of Her X-1 and to make a prediction of the polarization signal. We find that in the context of our model, the modulation of the cyclotron energy with phase is naturally explained by the fact that the emission from the column is relativistically beamed, and that different viewing angles on to the column and different parts of the column (where the gas has different velocities) dominate the emission in different phases (Falkner 2018). Several geometries and both the one-column and the two-column model can reproduce current observations, but we show that the polarization signal, especially the polarization angle, is very sensitive to the geometry.

\section{THE MODEL}

The emission from X-ray pulsars is difficult to model from first principles, as the picture is complicated by the presence of a strong magnetic field, by the importance of radiation pressure in the description of the accretion hydrodynamics and by the fact that the emitting gas is flowing with a high bulk velocity, up to half of the speed of light. Several attempts have been made to calculate the spectral formation in accreting X-ray pulsars based on theoretical models (Yahel 1980; Nagel 1981; Mészáros \& Nagel 1985a, b; Klein et al. 1996; Kawashima et al. 2016); however, the models often oversimplify some aspects of the radiation generation and propagation, and the results do not always agree very well with the observed spectral profiles. On the other hand, the procedure of fitting the spectra with multicomponent functions of energy as power laws, blackbodies, and exponential cut-offs is not easy to relate to the physical properties of the source.

The situation improved recently with the development by Becker and Wolff of a new model (hereafter the B\&W model) for the spectral formation (Becker \& Wolff 2005a, b, 2007; Wolff et al. 2016; West, Wolfram \& Becker 2017a, b). They were able to derive a spectrum from the solution of a coupled radiation and hydrodynamic transport equation that fits very well the observed profiles and returns estimates of the properties of the accretion flow, as for example, the optical thickness of the column, the temperature of the electrons, and the size of the column itself. Even though several simplifying assumptions are made to keep the treatment analytical, the $\mathrm{B} \& \mathrm{~W}$ model is the current theoretical model that best fits observations, which, however, does not provide any prediction on polarization.

In Paper I, we present a new method to model the polarized emission of accreting X-ray pulsars in the accretion column scenario, assuming that the structure of the column can be described as in the B\&W model. For the first time, our model takes into account the macroscopic structure and dynamics of the accretion column and the changes that happen during the propagation of the radiation towards the observer, including the effects of relativistic beaming, gravitational lensing, and quantum electrodynamics. We find that the beaming and polarization of the emission from the column are dominated by the presence of the strong magnetic field and by the high speed of the in-falling gas. Our calculations are exact and fully analytical and predict the polarization parameters of the radiation 


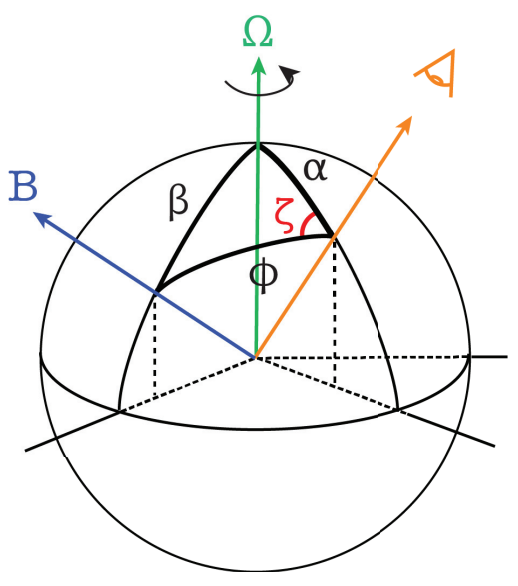

Figure 1. The geometry of the system is identified by the angle $\alpha$ between the line of sight (in orange) and the rotation axis (in green) and the angle $\beta$ between the rotation axis and the magnetic axis (in blue). The angle $\phi$ between the line of sight and the magnetic axis is the same as the phase in the case of an orthogonal rotator (see Paper I). The angle $\zeta$ (in red) between the magnetic axis and the rotation axis projected in the plane of the sky determines the polarization angle.

emitted by the accretion column independently of radiation transfer. The results depend on the physical parameters of the accretion column, in particular on the radius and height of the accretion column, on the strength of the magnetic field, on the velocity profile of the gas inside the column, and on the optical depth of the column. This information can be obtained by spectral fitting within the context of the B\&W model.

We here focus on Her X-1 because the accreting X-ray pulsar will be one of the main targets of the upcoming polarimeters and because its X-ray spectrum has been previously studied in the context of the B\&W model (Becker \& Wolff 2007; Wolff et al. 2016). Our model will also be applicable to predict the polarization signal of the emission from the accretion columns in different systems, including outbursting systems, whenever an X-ray spectrum is also available to constrain the physical parameters of the accretion columns. In this paper, we use the fit obtained by Wolff et al. (2016) of the phase-averaged NUSTAR spectrum of Her X-1. The parameters that we need for our model can be obtained from the following parameters in the paper:

(i) the radius of the accretion column $r_{0}=107 \mathrm{~m}$;

(ii) the observed cyclotron line energy $E_{\mathrm{cyc}}=37.7 \mathrm{keV}$;

(iii) the dimensionless parameter $\xi=1.36$, defined in equation (26) of Becker \& Wolff (2007), which determines the optical depth;

(iv) the accretion rate $\dot{M}=2.59 \times 10^{17} \mathrm{~g} \mathrm{~s}^{-1}$.

In addition, we employ a value of $M_{*}=1.5 \mathrm{M}_{\odot}$ for the mass and of $R_{*}=10 \mathrm{~km}$ for the radius of the neutron star, and a distance to the source of $6.6 \mathrm{kpc}$ (Reynolds et al. 1997). The inclination angle of Her X-1 and the angle between the rotation axis and the magnetic field axis can be somewhat constrained by analysing the pulse profile (see Section 3) but measuring the polarization angle as a function of phase will put much more stringent constraints on the geometry of the source (Section 5). Fig. 1 shows our convention for the names of the angles, where we have indicated the rotation axis $\hat{\Omega}$ in green, the magnetic axis in blue, and the line of sight in orange. The angle between the line of sight and the rotation axis

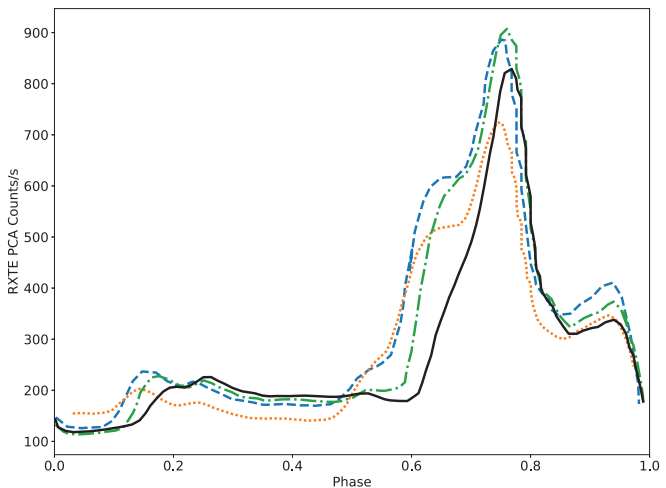

Figure 2. Pulse profiles averaged in the $9-13 \mathrm{keV}$ range from Vasco et al. (2013). They correspond to different phases in a 'Main On' of the 35-d period: 0.03 (orange dotted line), 0.10 (blue dashed line), 0.15 (green dash dotted line), and 0.2 (black solid line). The uncertainties are smaller than the line width.

$\alpha$ and the angle between the rotation axis and the magnetic axis $\beta$ remain constant as the star rotates, while the angle between the line of sight and the magnetic field axis $\phi$ and the angle between the magnetic axis and the rotation axis projected in the plane of the sky, $\zeta$, which determines the polarization angle, change with phase.

\section{PULSE PROFILE}

Vasco et al. (2013) analysed RXTE observations of a 'Main On' state of Her X-1, and they report the variation of spectral features with spin phase and of the pulse profile for four intervals in the precession period, those corresponding to phase $0.03,0.10,0.15$, and 0.2 of the 35-d period. In Fig. 2, we show the pulse profiles observed by Vasco et al. (2013, their fig. 2) averaged over the energy interval 9$13 \mathrm{keV}$. The main peak at about $\sim 0.75$ in pulse phase is surrounded by two 'shoulders': a left shoulder that shows strong variations with precession phase and that is almost absent in precession phase 0.2 (black solid line) and a right shoulder that only varies as much as the main peak with precession phase. Vasco et al. (2013) also report the variation of the intensity of the iron $\mathrm{K} \alpha$ line with pulse phase and precession phase: the intensity is quite constant with phase except in the peak, where it is undetectable, and in the left shoulder, where it is higher than average. Additionally, in the phases of the precession cycle where the left shoulder is less pronounced (like in phase 0.2 ), the peak in the iron line at the shoulder location is also less pronounced.

The changes in intensity of the main peak during the 'Main-On' are associated with the precession of the accretion disc: in the first phases, the disc gradually clears the view to the X-ray emitting region, and the flux increases, while towards the end of the 'MainOn', the inner edge of the disc starts blocking the emission region and the flux declines. The modulation in the left shoulder is much more pronounced than the modulation in the peak, and it does not follow the same trend. Moreover, while the iron line is undetectable at the main peak, in the left shoulder the line reaches its maximum intensity and it shares the same modulation with the left shoulder over the 35-d precession period. As the iron fluorescence is generated when an X-ray beam is reprocessed by cool material, this trend hints strongly that the left shoulder is coming from radiation reflected by the precessing disc. The left shoulder comes immediately before the main peak in time, which would suggest that it is caused by the reflection off the disc of the highly beamed emission from the 

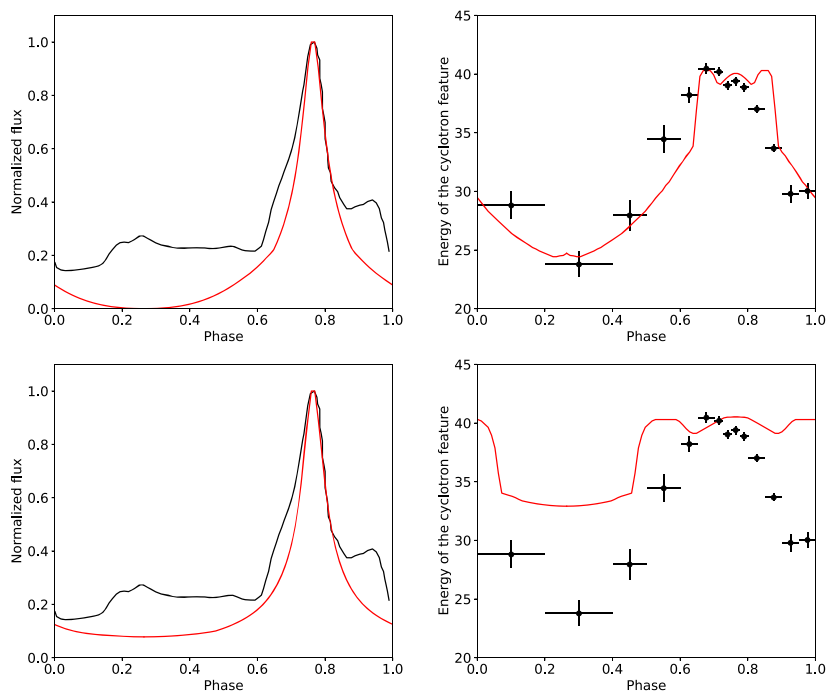

Figure 3. In the left-hand panels, in black, the observed pulse profile for Her $\mathrm{X}-1$ is shown for the phase in the precession cycle with the least contribution from the disc (phase 0.2, same as black solid line in Fig. 2) while in the righthand panels, the weighted mean values for the phase-dependent cyclotron line energy averaged over the 35-d precession period are plotted in black with error bars (data from Vasco et al. 2013). The solid red line shows the prediction for the one-column model for different geometries: in the top panels, $\alpha=83^{\circ}$ and $\beta=86^{\circ}$, while in the lower panels $\alpha=30^{\circ}$ and $\beta=155^{\circ}$.

column, pointing towards the disc right before pointing towards the observer. The right shoulder does not show a strong modulation with precession phase, and the strength of the iron line remains close to the average value in the phase of the right shoulder. Therefore, there is no particular indication whether the right shoulder is the result of a reflection off the disc or off the neutron star surface. It could be either or a combination of both.

We here assume that at the main peak, the emission from the accretion column, which is highly beamed, is pointing towards the observer, and that the varying emission in the phases outside of the main peak comes from reflections off the disc and/or the neutron star surface. We will discuss the effect on polarization of the two options in Section 5. Additionally, the disc direct emission could contribute, but we do not expect it to change with rotation phase and therefore it could only provide a constant background. Within our model, we can reproduce the pulse fraction and the shape of the peak both for the case in which the X-rays are emitted by two accretion columns at the magnetic poles of the neutron star or by a single column. There are several geometries, identified by the angles $\alpha$ and $\beta$ in Fig. 1, that produce a pulse profile that agrees with the observed one. The left-hand panels of Figs 3 and 4 show only some examples. In the left-hand panels of Fig. 3, the predicted pulse profile from the one-column model is shown in red for $\alpha=83^{\circ}$ and $\beta=86^{\circ}$ (upper panel) and for $\alpha=30^{\circ}$ and $\beta=155^{\circ}$ (lower panel), while in black we can see the pulse profile for the precession phase where the left shoulder is less pronounced (phase 0.2, also in black in Fig. 2). In the left-hand panels of Fig. 4, the predicted pulse profile from the two-column model is shown for $\alpha=50^{\circ}$ and $\beta=42^{\circ}$ (upper panel) and for $\alpha=75^{\circ}$ and $\beta=115^{\circ}$ (lower panel). We can see that in both figures, the different geometries can reproduce the pulse shape at the main peak, and the main difference between the geometries is how much the accretion column(s) contribute to the emission during the phases outside of the main peak.
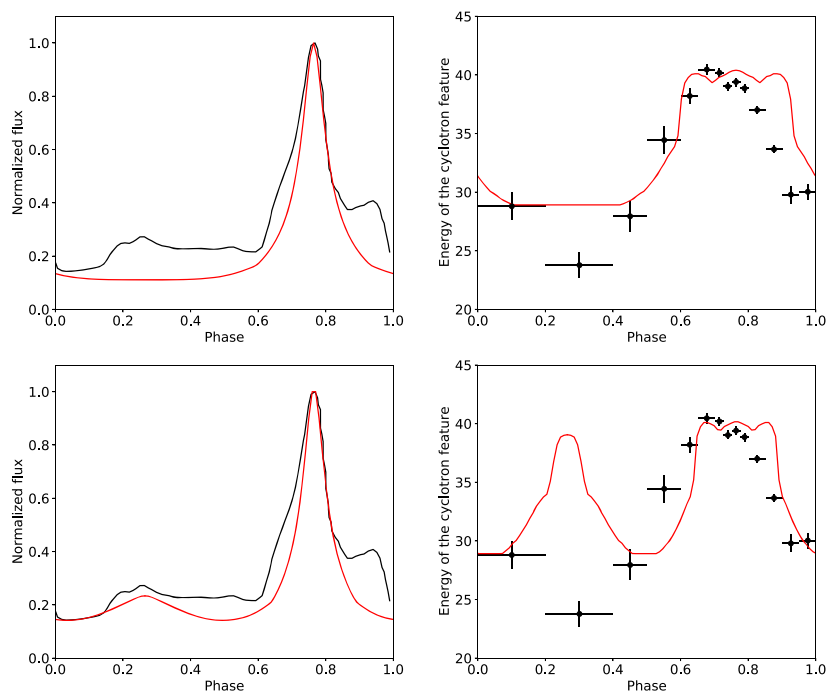

Figure 4. Same as in Fig. 3 but for the two-column model and different geometries: in the top panels, $\alpha=50^{\circ}$ and $\beta=42^{\circ}$, while in the lower panels $\alpha=75^{\circ}$ and $\beta=115^{\circ}$.

\section{CYCLOTRON FEATURE}

Vasco et al. (2013) and Fürst et al. (2013) analysed the evolution of the centroid energy of the CRSF in Her X-1 using data from RXTE and NUSTAR. They both found that the cyclotron line energy follows roughly the pulse profile, with an almost sinusoidal shape, and that there is no indication for a modulation over the $35-\mathrm{d}$ period. The variation in cyclotron energy with phase, which has been observed in may X-ray pulsars, has been previously explained by the fact that as the star rotates, we see radiation coming from different hotspots on the surface of the neutron star where the strength of the magnetic field is different (e.g. in Kreykenbohm et al. 2004, and references therein). In our model, the modulation of the line centroid with phase is naturally explained by the fact that the scattering electrons in the accretion column are highly relativistic. In fact, at the top of the column, the ionized gas is moving at about half the speed of light, and the radiation emitted by the column is highly beamed towards the surface of the star. For this reason, the main peak in the pulse profile corresponds to when one of the accretion columns is almost behind the star ( $\phi$ is close to $\pi$ in Fig. 1) and the strong beaming causes the cyclotron energy to be shifted to higher values. As the star rotates, we see the column at smaller angles, and therefore the beaming is less intense and the cyclotron line energy decreases.

The right-hand panels of Figs 3 and 4 show the weighted mean values for the phase-dependent cyclotron line energy averaged over the 35-d precession period in black with error bars, as measured by Vasco et al. (2013, their fig. 5), together with the predictions of our model, in red, for the same geometries described in Section 3. In the upper right panel of Fig. 3, which is the case of an almost orthogonal rotator with one column, the modulation in cyclotron energy predicted by the model seems to best reproduce the observed one. In the lower panel of the same figure, the single column is always at a high angle $\phi$ with respect to the observer, and therefore the effect of the beaming on the CRSF energy is always important, reducing the modulation with phase. Fig. 4 shows the two-columns case, in which the contribution from the second column increases the minimum in the cyclotron line energy in the upper right panel and even produces a second peak in the lower right panel. It is worth 

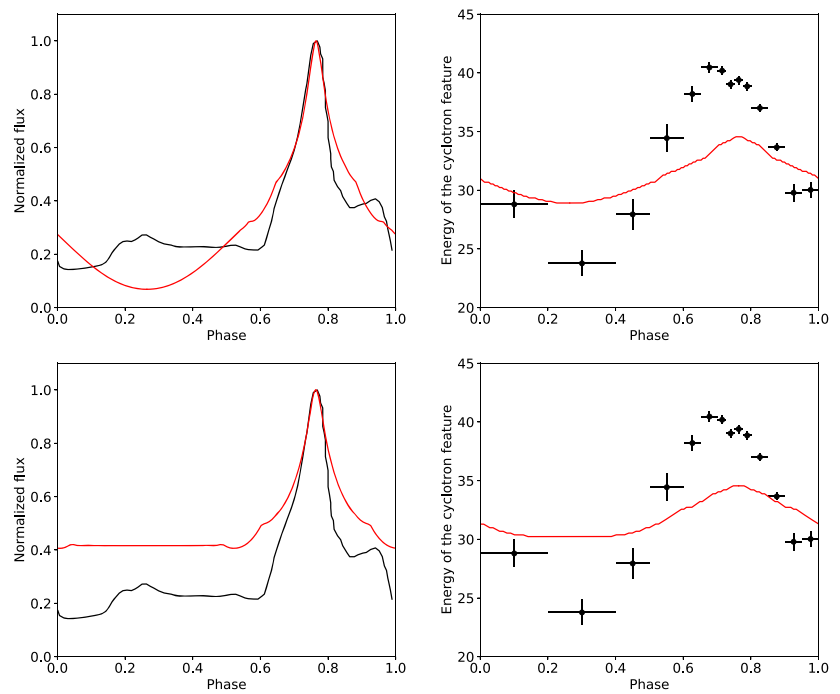

Figure 5. Same as in Fig. 3 but for the short one-column model with $\alpha=$ $67^{\circ}$ and $\beta=120^{\circ}$ (upper panels) and for the short two-columns model with $\alpha=46^{\circ}$ and $\beta=40^{\circ}$ (lower panels).

mentioning that we have not adjusted our model in any way to meet the data, and that these predictions simply come from the value of the cyclotron line estimated in Wolff et al. (2016) and from choosing the geometry that best reproduces the main peak in flux.

Figs 3 and 4 only show the prediction for the emission from the column in red. If part of the emission in the phases outside the main peak is coming from the neutron star surface, for example, because of reflection or reprocessing, the modulation of the cyclotron line energy can be affected by the fact that in the reflecting region the magnetic field has a different strength than in the column. For example, if the right shoulder is indeed caused by a reflection on the surface, away from the magnetic pole, the local magnetic field would be lower than in the column and this could explain why the observed cyclotron energy declines faster with phase after the main peak in the observations compared to the model prediction. For the same reason, the fact that the two-column model fails to reproduce the minimum in the cyclotron energy modulation could be due to the fact that at that phase, the emission from the column is a small contribution to the total emission and that reflections from the surface could be dominating the emission. An indication could come from the width of the cyclotron feature: when different regions where the magnetic field strength has different values contribute to the emission, we expect the cyclotron feature to be wide. Vasco et al. (2013) could not resolve the width of the cyclotron feature on the $35-\mathrm{d}$ period because of large scatter in the data, but they found that on average the cyclotron feature is wider in the phases between 0.0 and 0.4 .

In Paper I, we analysed the possibility of the accretion column being shorter than what is predicted by the $\mathrm{B} \& \mathrm{~W}$ model. In particular, with the parameters obtained by Wolff et al. (2016) for Her X-1, the $\mathrm{B} \& \mathrm{~W}$ model predicts a column height $z_{\max }=6.6 \mathrm{~km}$. If a different assumption is made on the velocity profile of the in-flowing gas and on the nature of the shock at the top of the column, the height of the column could be different. For example, in Paper I we analysed the case in which an adiabatic shock at the top of the column drastically reduces the speed of the incoming gas, reducing also the height of the column to about $1.4 \mathrm{~km}$. The predictions of the short-column model are shown in Fig. 5 for the one- and the two-column case in the geometries that more closely reproduce the pulse profile. In the shorter column model, the scattering electrons are moving much slower, and therefore the relativistic beaming is less pronounced. For this reason, the short-column model fails to reproduce both the narrow peak observed in the pulse profile of Her X-1 and the strong modulation of the cyclotron line energy. We therefore conclude that, even if the accretion column could in principle be shorter than what is predicted by the $\mathrm{B} \& \mathrm{~W}$ model, it cannot be too short, or conversely, the speed of the electrons cannot be too low, or the model fails to reproduce the observed profiles in flux and cyclotron energy.

\section{POLARIZATION SIGNAL}

From the analysis of the pulse profile and of the CRSF, we see that the models that best reproduce the observed profiles are the one-column model with $\alpha=83^{\circ}$ and $\beta=86^{\circ}$ and the two-column model with $\alpha=50^{\circ}$ and $\beta=42^{\circ}$ (or equivalent geometries). We now analyse what is the polarization signal that we expect from these models. The upper panels of Figs 6 and 7 show the polarization parameters, polarization fraction and angle, of the emission from the column(s) as a function of phase for different photon energies. In both models, the polarization fraction (in blue) is very high at low energy and reaches a broad peak in the phase that coincides with the narrower peak in flux (in black). The main difference in polarization fraction between the two models is that the one-column model has a deep minimum in the antiphase of the peak, while the polarization fraction in the two-column model is quite flat outside the peak.

As expected, the polarization angle (in red) is quite different between the two models. In both models, below $10 \mathrm{keV}$, the light is polarized in the ordinary mode, so the polarization direction coincides with the direction of the magnetic field in the sky. In the one-column model, the polarization angle has a swing coincidentally with the peak because the column is going behind the star, and therefore the polarization angle swings from $\zeta \sim 90^{\circ}$ to $\zeta \sim-90^{\circ}$. The fact that $\alpha$ is close to $90^{\circ}$ means that we see an additional, very sharp swing at the antiphase of the main peak. For the two-column model, in the particular geometry that we picked, the swing in the peak only goes between $\zeta \sim-60^{\circ}$ and $\zeta \sim 60^{\circ}$ because $\beta$ is lower, and the swing at the antipeak is much smoother because $\alpha$ is also lower.

In both models, the emission at $30 \mathrm{keV}$, close to the cyclotron energy, is polarized in the direction perpendicular to the magnetic axis (in the extraordinary mode) and therefore the polarization angle follows the same pattern as at low energy, only shifted by $90^{\circ}$. At $20 \mathrm{keV}$, the polarization is in the ordinary mode in the peak (around 0.75 in phase), but it goes through a zero and changes to the extraordinary mode at phases $\sim 0.7$ and $\sim 0.85$, which results in a shift of the polarization angle of $90^{\circ}$ outside the peak.

The lower panels of Figs 6 and 7 show the comparison between the polarization fraction calculated without including the quantum electrodynamics (QED) effect of vacuum birefringence (orange dash-dotted line) and including QED (blue dashed line). We can see that the effect of QED is mainly to reduce the polarization fraction everywhere and especially outside of the peak. More details can be found in Paper I.

Figs 6 and 7 only depict the emission coming from the accretion column(s). As discussed in Section 3, outside of the peak we expect a large contribution to come from either the accretion disc or the surface of the neutron star. If the disc itself is emitting in the energy range of interest, such emission should stay constant with rotation phase and show variability with the $35-\mathrm{d}$ period. A direct contribution from the disc would dilute the polarization shown in Figs 6 and 7 because the emission from an accretion disc is expected to be weakly polarized, with a peak of about 11 per cent parallel to 

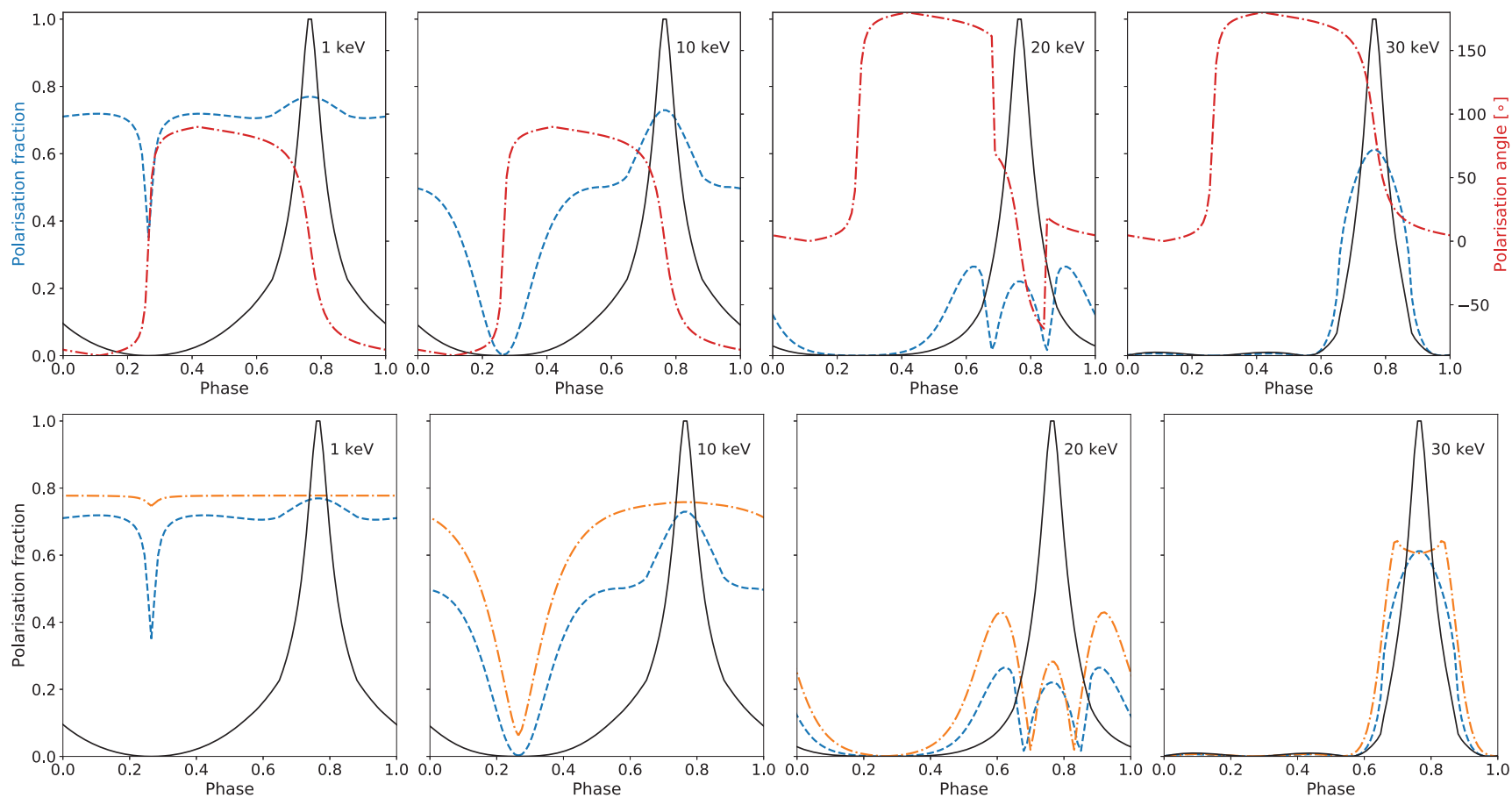

Figure 6. Polarization parameters for the emission from the column in the one-column model with $\alpha=83^{\circ}$ and $\beta=86^{\circ}$ as function of phase and for four photon energies at the observer, from left to right: 1, 10, 20, and $30 \mathrm{keV}$. Upper panels: polarization fraction (blue dashed line) and polarization angle (red dash-dotted line). Lower panels: polarization fraction including the effect of QED (blue dashed line, same as upper panels) and without QED (orange dash-dotted line).
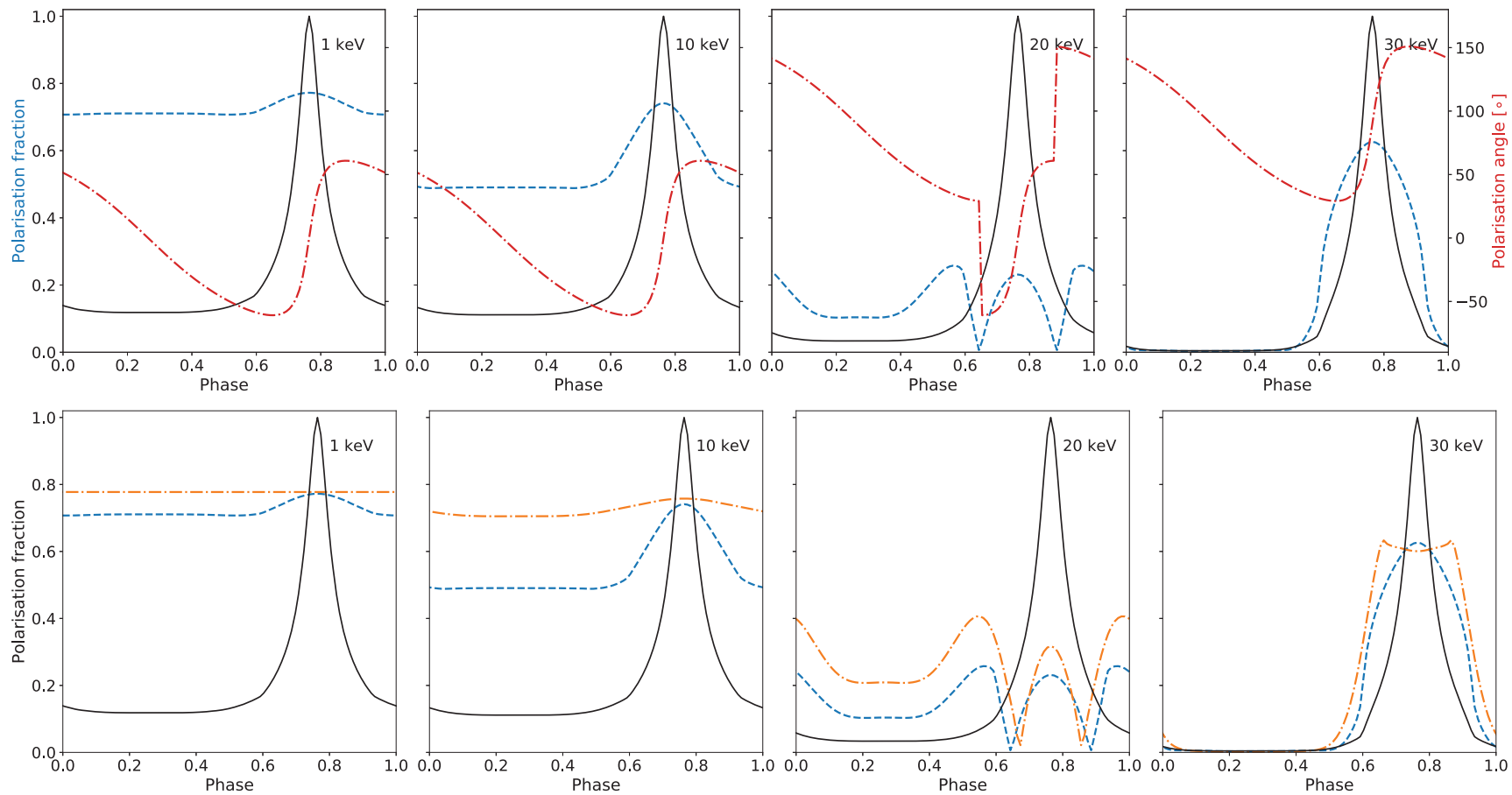

Figure 7. Same as Fig. 6 but for the total emission from the columns in the two-columns model with $\alpha=50^{\circ}$ and $\beta=42^{\circ}$.

the disc plane if the disc is seen edge-on (e.g. Chandrasekhar 1960; Davis et al. 2009; Schnittman \& Krolik 2009, 2010; Caiazzo \& Heyl 2018, and references therein). Although direct emission from the disc would stay constant with phase, a reflection component could instead change with phase. For example, we suggested in Section 3 that the 'left shoulder' in the pulse profile could be caused by the disc reflecting the beamed X-ray emission from the accretion column. In this case, we expect the scattered radiation to be polarized in the same direction as the pulse and to be highly polarized. If reflected emission from the disc contributes to phases far from the main pulse, we expect it to be polarized perpendicular to the disc. 
The 'right shoulder' in the pulse profile could come from either a reflection off the disc or off the neutron star surface. As we have shown in Paper I, the radiation from the accretion column is beamed downward by special relativity and bent downward towards the stellar surface by gravity. We therefore expect a substantial portion of the surface to be illuminated by the column. Depending on whether the heat is deposited deep inside the stellar surface or if the illuminating photons are scattered in the upper layers of the atmosphere, the expected polarization signal is quite different. In the former case, we expect the average energy of the emission in the 'right shoulder' to be less than near the peak, the polarization to be mainly in the Xmode for low energies, and the polarization fraction to decrease with energy (see Caiazzo, Heyl \& Turolla 2019, and references therein). In the latter case, the illuminating photons are scattered and possibly boosted in energy through Comptonization off the surface and therefore we expect the average energy of the emission to be higher than or similar to the one near the peak. The polarization signal would be also very different, as the scattered photons would be polarized mainly in the O-mode (see Mészáros \& Nagel 1985b, and Paper I).

If the neutron star is precessing with a 35-d period, as suggested by some authors (Brecher 1972; Truemper et al. 1986; Postnov et al. 2013), we expect the polarization direction both for the main emission and the reflected emission to shift with the precession period.

\section{CONCLUSIONS}

We have presented a model for the polarized emission from one or two accretion columns in the X-ray pulsar Her X-1. This model is able to reproduce the pulse fraction and the pulse shape of the main peak in the pulse profile of Her X-1. Additionally, the observed modulation with rotational phase of the cyclotron energy is naturally reproduced by some geometries in our model that also reproduce the pulse profile. The model is built upon the B\&W model (see Paper I) for the phase-averaged spectrum for Her X-1, so it also is able to satisfactorily reproduce the current observations and to build upon these to make clear predictions of the expected polarization signal. Observations of the polarized signal will help constraining the geometry of the system, in particular the nature of the 35-d superorbital period, by determining if the disc or the neutron star precess, or both. Furthermore, polarization will help decide the nature of the emission outside the main pulse that still varies periodically with pulsar period, specifically if it is reprocessed by the disc or the stellar surface, and in the latter case, how deeply the energy is deposited in the neutron star's atmosphere. The dawn of astrophysical $\mathrm{X}$-ray polarimetry will dramatically enhance our understanding of $\mathrm{X}$-ray pulsars in general and Her X-1 in particular.

\section{ACKNOWLEDGEMENTS}

We would like to thank Michael Wolff, Peter Becker, and the XMAG collaboration for valuable input, and Sterl Phinney for useful comments. The research was supported by NSERC Canada, Compute Canada, a Burke Fellowship at Caltech, and a Four-Year Fellowship at UBC.

\section{DATA AVAILABILITY}

The values for the predicted polarization fraction and angle as function of phase and energy displayed in Figs 6 and 7 can be reproduced following the equations introduced in Paper I, and are also available in the public Github repository https://github.com/U BC-Astrophysics/QEDSurface/tree/master/Caiazzo_Heyl_Model.

\section{REFERENCES}

Araya-Góchez R. A., Harding A. K., 2000, ApJ, 544, 1067

Becker P. A., Wolff M. T., 2005a, ApJ, 621, L45

Becker P. A., Wolff M. T., 2005b, ApJ, 630, 465

Becker P. A., Wolff M. T., 2007, ApJ, 654, 435

Becker P. A. et al., 2012, A\&A, 544, A123

Beilicke M. et al., 2014, J. Astron. Instrum., 3, 1440008

Boynton P. E., Crosa L. M., Deeter J. E., 1980, ApJ, 237, 169

Brecher K., 1972, Nature, 239, 325

Caiazzo I., Heyl J., 2018, Phys. Rev. D, 97, 083001

Caiazzo I., Heyl J., Turolla R., 2019, Astrophysics and Space Science Library, Vol. 460, Polarimetry of Magnetars and Isolated Neutron Stars. Springer Nature, Switzerland, AG, p. 301

Caiazzo I., Heyl J., 2020, MNRAS, available at: https://doi.org/10.1093/mn ras/staa3429

Chandrasekhar S., 1960, Radiative Transfer. Dover, New York

Chauvin M. et al., 2018, MNRAS, 77, L45

Choi C. S., Nagase F., Makino F., Dotani T., Kitamoto S., Takahama S., 1994, ApJ, 437, 449

Davis S. W., Blaes O. M., Hirose S., Krolik J. H., 2009, ApJ, 703, 569

Deeter J. E., Scott D. M., Boynton P. E., Miyamoto S., Kitamoto S., Takahama S., Nagase F., 1998, ApJ, 502, 802

Falkner S., 2018, PhD thesis, Friedrich-Alexander-Universität ErlangenNürnberg

Feng H. et al., 2019, Exp. Astron., 47, 225

Feng H. et al., 2020, Nat. Astron., 4, 511

Fürst F. et al., 2013, ApJ, 779, 69

Gaenther H. M., Egan M., Heilmann R. K., Heine S. N. T., Hellickson T., Frost J., Marshall H. L., Schulz N. S., Theriault-Shay A., 2017, in O’Dell S. L., Pareschi G., eds, Proc. SPIE Conf. Ser. Vol. 10399, Optics for EUV, X-Ray, and Gamma-Ray Astronomy VIII. SPIE, Bellingham, p. 13

Gerend D., Boynton P. E., 1976, ApJ, 209, 562

Giacconi R., Gursky H., Kellogg E., Levinson R., Schreier E., Tananbaum H., 1973, ApJ, 184, 227

Jahoda K. et al., 2019, Bull. Am. Astron. Soc., 51, 181

Ji L., Staubert R., Ducci L., Santangelo A., Zhang S., Chang Z., 2019, MNRAS, 484, 3797

Kawashima T., Mineshige S., Ohsuga K., Ogawa T., 2016, PASJ, 68, 83

Klein R. I., Arons J., Jernigan G., Hsu J. J. L., 1996, ApJ, 457, L85

Klochkov D. et al., 2008, A\&A, 482, 907

Krawczynski H. et al., 2019, Bull. Am. Astron. Soc., 51, 150

Kreykenbohm I., Wilms J., Coburn W., Kuster M., Rothschild R. E., Heindl W. A., Kretschmar P., Staubert R., 2004, A\&A, 427, 975

Leahy D. A., 2002, MNRAS, 334, 847

Lutovinov A. A., Tsygankov S. S., Suleimanov V. F., Mushtukov A. A., Doroshenko V., Nagirner D. I., Poutanen J., 2015, MNRAS, 448, 2175

Mészáros P., Nagel W., 1985a, ApJ, 298, 147

Mészáros P., Nagel W., 1985b, ApJ, 299, 138

Mushtukov A. A., Tsygankov S. S., Serber A. e. V., Suleimanov V. F., Poutanen J., 2015, MNRAS, 454, 2714

Mushtukov A. A., Verhagen P. A., Tsygankov S. S., van der Klis M., Lutovinov A. A., Larchenkova T. I., 2018, MNRAS, 474, 5425

Nagel W., 1981, ApJ, 251, 278

Nishimura O., 2014, ApJ, 781, 30

Postnov K., Shakura N., Staubert R., Kochetkova A., Klochkov D., Wilms J., 2013, MNRAS, 435, 1147

Poutanen J., Mushtukov A. A., Suleimanov V. F., Tsygankov S. S., Nagirner D. I., Doroshenko V., Lutovinov A. A., 2013, ApJ, 777, 115

Reynolds A. P., Quaintrell H., Still M. D., Roche P., Chakrabarty D., Levine S. E., 1997, MNRAS, 288, 43

Schnittman J. D., Krolik J. H., 2009, ApJ, 701, 1175

Schnittman J. D., Krolik J. H., 2010, ApJ, 712, 908

Scott D. M., Leahy D. A., 1999, ApJ, 510, 974 
Scott D. M., Leahy D. A., Wilson R. B., 2000, ApJ, 539, 392

She R. et al., 2015, in Siegmund O. H., ed., Proc. SPIE Conf. Ser. Vol. 9601, UV, X-Ray, and Gamma-Ray Space Instrumentation for Astronomy XIX. SPIE, Bellingham, p. 96010I

Staubert R., Klochkov D., Vasco D., Postnov K., Shakura N., Wilms J., Rothschild R. E., 2013, A\&A, 550, A110

Staubert R., Klochkov D., Wilms J., Postnov K., Shakura N. I., Rothschild R. E., Fürst F., Harrison F. A., 2014, A\&A, 572, A119

Tananbaum H., Gursky H., Kellogg E. M., Levinson R., Schreier E., Giacconi R., 1972, ApJ, 174, L143

Truemper J., Pietsch W., Reppin C., Voges W., Staubert R., Kendziorra E., 1978, ApJ, 219, L105

Truemper J., Kahabka P., Oegelman H., Pietsch W., Voges W., 1986, ApJ, 300, L63

Tsygankov S. S., Lutovinov A. A., Churazov E. M., Sunyaev R. A., 2006, MNRAS, 371,19
Vasco D., Staubert R., Klochkov D., Santangelo A., Shakura N., Postnov K., 2013, A\&A, 550, A111

Weisskopf M. C. et al., 2016, in den Herder J.-W. A., Takahashi T., Bautz M., eds, Proc. SPIE Conf. Ser. Vol. 99005, Space Telescopes and Instrumentation 2016: Ultraviolet to Gamma Ray. SPIE, Bellingham, p. 990517

West B. F., Wolfram K. D., Becker P. A., 2017a, ApJ, 835, 129

West B. F., Wolfram K. D., Becker P. A., 2017b, ApJ, 835, 130

Wolff M. T. et al., 2016, ApJ, 831, 194

Yahel R. Z., 1980, ApJ, 236, 911

Zhang S. N. et al., 2016, in den Herder J.-W. A., Takahashi T., Bautz M., eds, Proc. SPIE Conf. Ser. Vol. 9905, Space Telescopes and Instrumentation 2016: Ultraviolet to Gamma Ray. SPIE, Bellingham, p. 99051Q

This paper has been typeset from a $\mathrm{T}_{\mathrm{E}} \mathrm{X} / \mathrm{L} \mathrm{T}_{\mathrm{E}} \mathrm{X}$ file prepared by the author. 